\title{
A Novel Technique for Low-Cost Embedded Non-intrusive Fiber Monitoring of P2MP Optical Access Networks
}

\author{
W. Chen, B. De Mulder, J. Vandewege, X.Z. Qiu, J. Bauwelinck and B. Baekelandt \\ Ghent University, INTEC/IMEC, Sint-Pietersnieuwstraat 41, 9000 Gent, Belgium. \\ wei.chen@intec.ugent.be
}

\begin{abstract}
A low cost embedded OTDR method is presented for monitoring the fiber plant of TDM optical networks without extra optical components nor performance penalty. It localizes abrupt changes in link attenuation without disturbing network operation. (C) 2007 Optical Society of America

OCSIS codes: (060.4250) Networks; (060.2330) Fiber optics communications
\end{abstract}

\section{Introduction}

More and more TDM broadband optical access networks are deployed with a passive optical network (PON) architecture, where an optical line termination (OLT) connects to a number of optical network units (ONUs) via fiber and power splitters. Time slots are granted to each ONU for sending bursts of upstream data towards the OLT in a time division multiple access (TDMA) scheme, and ONU Laser Diode (LD) and laser driver operate in burst mode on a nanosecond scale. In such complex networks, it is difficult to perform measurements on the fiber plant without disturbing the many ongoing services. Conventionally, fiber monitoring is performed by optical timedomain reflectometry (OTDR) measurements at the OLT side in an intrusive way. OLT OTDR data however suffer from reduced sensitivity due to high splitting losses and from ambiguous diagnosis of fiber link failure due to the superposition of OTDR traces originating from different fiber branches. A recent idea about low-cost embedded OTDR suitable for ONU monitoring reuses the LD and laser driver which are present at the fiber endpoint for data transmission [1]. This approach can reduce the cost to a certain extent because no dedicated OTDR laser and driver are needed, but an additional coupler and photodetector are still needed.

This paper presents a novel, low-cost technique, called FiberMon, for embedded non-intrusive fiber plant monitoring. The OTDR functionality is purely electronic, and can be integrated into any Fabry-Pérot (FP) or Vertical-Cavity Surface-Emitting Lasers (VCSEL) laser transmitter without optical isolator. O/E conversion of the OTDR signal is performed by the data LD itself, and/or by a back-facet monitor photodiode (MPD) contained in the laser module. As FiberMon relies on the upstream data bursts for fiber excitation, it does not require transmission of specific OTDR signals. FiberMon can provide round the clock data on the health status of the fiber plant, and trigger preventive actions without disturbing ongoing traffic nor penalizing the network performance.

\section{Embedded non-intrusive fiber monitoring (FiberMon)}

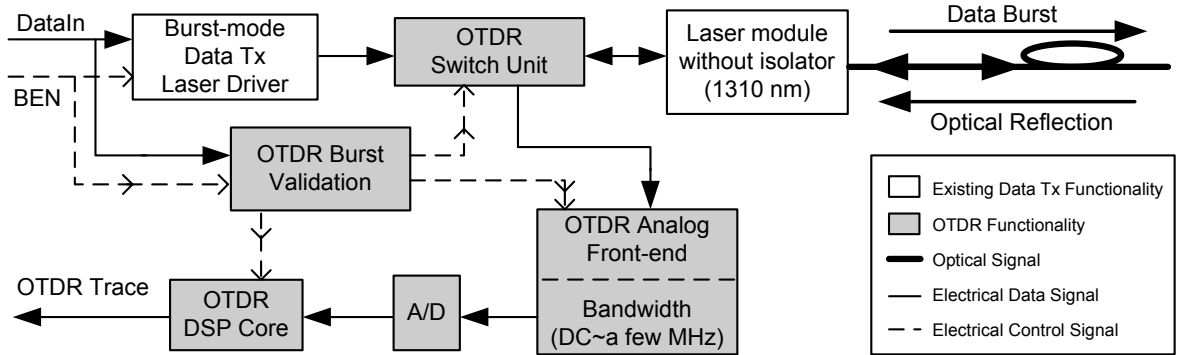

Fig. 1. ONU burst-mode data transmitter with embedded non-intrusive OTDR functionality

FiberMon was designed to be compatible with Gigabit PON (GPON) specifications [2-3], but can be used in a wide range of fiber networks provided that, after transmission of a data burst, suitable time windows can be found for acquiring the backscattering signal. The block diagram of an ONU burst-mode transmitter (BM-TX) with embedded non-intrusive OTDR functionality (Fig. 1) shows a traffic data input (DataIn), a burst transmission enable (BEN) signal and an OTDR trace output. A $1310 \mathrm{~nm}$ laser module with MPD generates data bursts and the MPD monitors the emitted optical power. When the BEN signal indicates that no data bursts are transmitted upstream (idle window), the optical front-end is switched from transmit mode into OTDR mode, and the LD (switched from 


\section{OThE4.pdf}

forward-bias to low or zero-bias) and/or the MPD act as an OTDR photodetector. So the backscattering caused by the data burst can be acquired with maximal reuse of components and at minimal cost. The data bursts transmitted by the ONU have a variable length and a limited optical power compliant with GPON specifications, and cannot be used as an excitation for classic impulse-response OTDR. However, from known parameters, the data burst length $\left(\mathrm{W}_{\text {Burst, }} \mu \mathrm{s}\right.$ scale) and the length of the fiber to be tested $\left(\mathrm{L}_{\text {Fiber }}, \mathrm{km}\right)$, the BM-TX can detect whether a data burst is long enough as excitation for step-response OTDR. If this happens, the OTDR measurement only depends on the fiber status and the ONU excitation power, and not on the data burst length. This negative step response (NSR) approach provides a novel way to reuse traffic data bursts to perform truly non-intrusive fiber monitoring.

The OTDR analog front-end (OAF) bandwidth is limited to a few MHz, trading-off spatial resolution and noise reduction. The high bit rate PON data bursts up to $1.25 \mathrm{Gbps}$ add a limited amount of noise to the optical reflections inside the OTDR bandwidth, but this noise power decreases at higher data rates. An OTDR burst validation (OBV) circuit identifies data bursts that are suitable for generating an NSR, and also checks whether the idle window ( $T_{\text {Idle }}$ ) is long enough to capture a full OTDR trace. As data transmission has priority over OTDR operation, the OTDR switch unit (OSU) switches the BM-TX functionality from OTDR mode back to transmit mode when a new data burst is to be sent, in a way that is fully transparent for all MAC (medium access control) functions. This procedure is repeated for every obtained NSR, to improve the knowledge of the fiber status. Assuming that the speed of light inside the fiber is $5 \mu \mathrm{s} / \mathrm{km}$, the condition for a valid burst to yield a complete NSR, covering the full fiber length, is straightforward:

$$
\left(W_{\text {Burst }} \text { and } T_{\text {Idle }}\right) \geq L_{\text {Fiber }} * 10(\mu \mathrm{s})
$$

In case $\mathrm{W}_{\text {Burst }}$ is smaller than $\mathrm{L}_{\text {Fiber }}{ }^{*} 10$, a pulse response can be obtained. Mathematic analysis has proven that a pulse response with arbitrary width can be converted to the standard NSR when the OTDR front-end is sufficiently linear [4]. So shorter data bursts can also yield OTDR measurements after suitable post-processing.

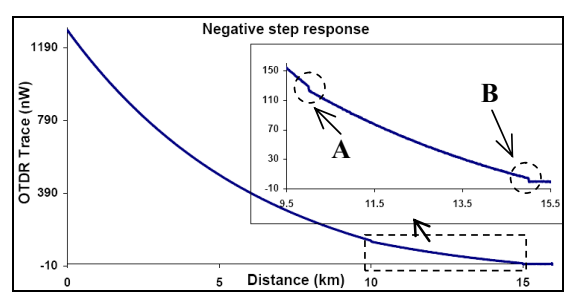

Fig. 2. Simulation of the

negative step response in linear scale

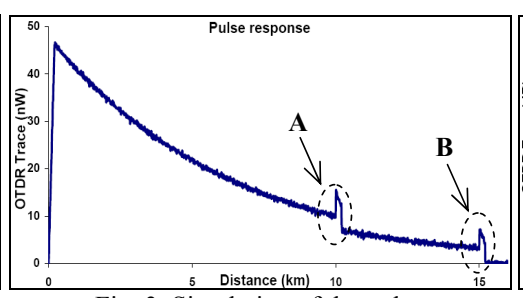

Fig. 3. Simulation of the pulse

$(2 \mu \mathrm{s})$ response in linear scale

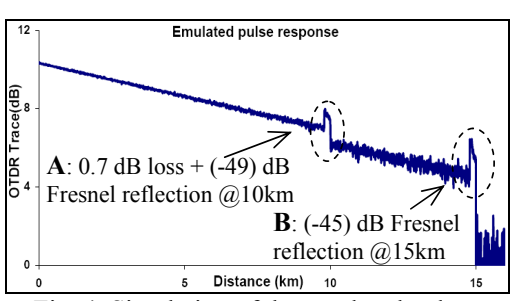

Fig. 4. Simulation of the emulated pulse

$(2 \mu \mathrm{s})$ response in log scale

Simulations were performed to compare fiber pulse response and NSR. The fiber under test consists of two sections of ITU-T G.652 compliant single-mode fiber with similar backscattering constant and attenuation, $10 \mathrm{~km}$ and $5 \mathrm{~km}$ long. A combined $0.7 \mathrm{~dB}$ loss and $-49 \mathrm{~dB}$ Fresnel reflector is caused by a fiber interconnection (A) and another $-45 \mathrm{~dB}$ Fresnel reflection (B) is present at the end. The fiber is excited with respectively a $2 \mu$ s pulse and a $150 \mu$ s step $\left(\mathrm{W}_{\text {Burst }}\right)$. Given $5 \mathrm{MHz}$ OAF bandwidth and $3 \mathrm{dBm}$ injected optical power, the OTDR traces show the optical artifacts A and B at 10 resp. 15 km (Fig. 2 to Fig. 4). Fig. 2 and 3 use a linear scale. For comparison, Fig. 4 shows an emulated pulse response derived from the NSR by subtracting its shifted version with emulated pulse width $2 \mu \mathrm{s}$. The exponentially decaying NSR signal of Fig. 2, caused by the backscatter of fiber sections filled completely with light, is quite strong compared to the pulse response of Fig. 3. In the NSR curve, a discrete Fresnel reflection does not show up as a reflected pulse but as a step in the level of the reflected light, which can increase the spatial resolution substantially. The step size is similar to the pulse height in the pulse response, meaning that the SNR of the Fresnel reflection is similar as with pulse response.

\section{Experimental results}

Different optical components were made to act as an OTDR photodetector: an FP laser module with integrated LD/MPD (FP-LD/FP-MPD), a VCSEL sample kindly provided by Vertilas and, as a reference, the more expensive combination of an extra PIN photodiode and a 10/90 optical coupler. Table 1 lists some measured parameters of the OTDR RX with $5 \mathrm{MHz}$ bandwidth. Due to higher responsivity, the FP-LD and VCSEL show better RX sensitivity than the FP-MPD. Measurements at room temperature indicate that the responsivity of the zero-biased VCSEL is polarization independent, while the zero-biased FP-LD as well as the FP-MPD show some polarization dependency. By simultaneously measuring on both the FP-LD and the FP-MPD however, a relatively stable combined optical reflection is obtained, and the polarization dependency of a FP laser module can be compensated considerably. 


\section{OThE4.pdf}

Table 1 Comparison of OTDR RX

\begin{tabular}{|c|c|c|}
\hline $\begin{array}{c}\text { Different acquisition } \\
\text { approaches }\end{array}$ & $\begin{array}{c}\text { Responsivity } \\
(\mathrm{A} / \mathrm{W})\end{array}$ & $\begin{array}{c}\text { Sensitivity } \\
(\mathrm{dBm})\end{array}$ \\
\hline $\begin{array}{c}\text { FP-LD } \\
\text { (Sumitomo SLT4716) }\end{array}$ & 0.12 & -48.0 \\
\hline $\begin{array}{c}\text { FP-MPD } \\
\text { (Sumitomo SLT4716) }\end{array}$ & 0.033 & -43.6 \\
\hline $\begin{array}{c}\text { VCSEL } \\
\text { (Vertilas) }\end{array}$ & 0.178 & -49.7 \\
\hline $\begin{array}{c}\text { 10/90 Coupler+PIN } \\
\text { (Siemens SFH2223) }\end{array}$ & 0.079 & -49.4 \\
\hline
\end{tabular}

Fig. 5 shows an experimental setup, built to test the proposed FiberMon technique with FP-LD, and to validate the presented simulations. An SC-SC connection with $0.7 \mathrm{~dB}$ optical loss and $-49 \mathrm{~dB}$ Fresnel reflector emulates a poor fiber splice between two sections of G.652 fiber. Another $-45 \mathrm{~dB}$ Fresnel reflection at the end is caused by an optical power splitter. The BM-TX transmits high bit-rate, $150 \mu \mathrm{s}$ data bursts with $4 \mathrm{dBm}$ peak optical power, and the embedded OTDR unit captures the fiber backscattering after transmission. Substantial averaging, which may be performed at low speed by an off-chip microcontroller, yields the measured NSR OTDR trace shown in Fig. 6. Postprocessing the NSR OTDR trace reveals the aforementioned artifacts. The emulated pulse OTDR trace (in Fig. 7) is found by converting the measured NSR curve, and illustrates the fiber plant artifacts in a more familiar way. Such conversion however is not necessary for exploiting the NSR OTDR method. The transient, and the ripple occurring at short distance from the source, are due to speed limitations and parasitics of the board-level electronics used to perform the switch-over from transmit to OTDR mode. This can be improved substantially by integration of the high-speed electronics inside the burst-mode laser driver chip. Such integration can be performed at a marginal cost, as it requires very little chip area compared to the large on-chip devices handling the substantial laser drive currents.

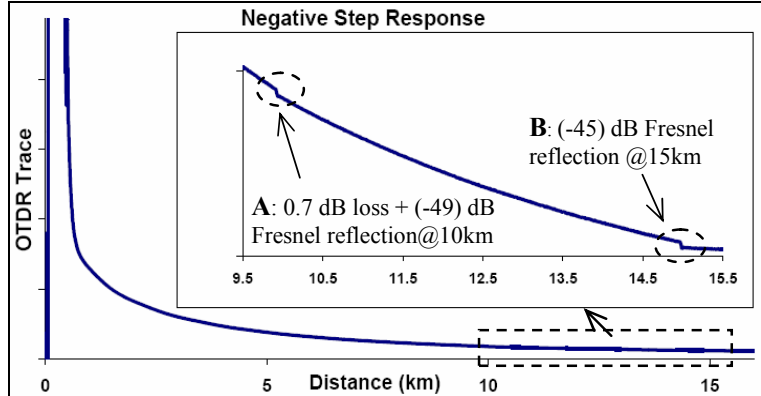

Fig. 6. Measured NSR OTDR trace on FP-LD in linear scale

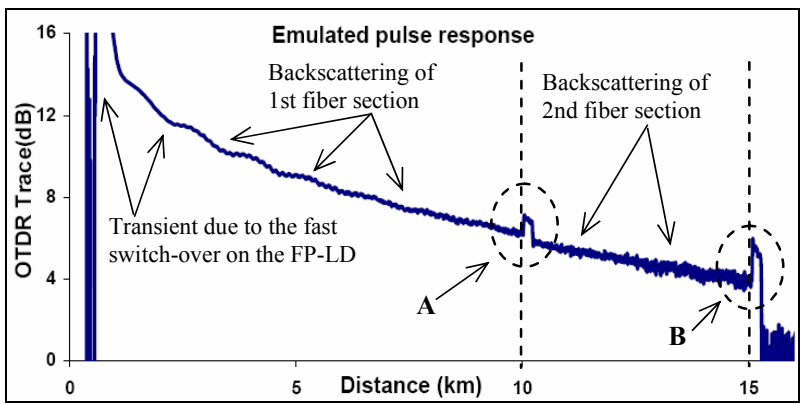

Fig. 7. Emulated pulse $(2 \mu \mathrm{s})$ OTDR trace on FP-LD in log scale

\section{Conclusion}

Embedded non-intrusive fiber plant monitoring can be performed from a laser data transmitter without any penalty on network performance and without interference with the MAC. FP or VCSEL transmitters do not need additional optical components, but only a limited amount of high-speed electronics to be integrated in the laser driver chip at marginal cost. No transmission of specific OTDR signals is required, and the required signal processing can be handled during idle time of an off-chip microcontroller. Information on the state of the fiber plant can be obtained continuously, and used to prove fiber plant performance, or to trigger preventive maintenance or repair actions. So fiber plant degradation can be detected long before transmission errors occur or services fail. Simulations and experimental results show that NSR OTDR can yield as good a precision for locating an abrupt change in attenuation as the classic impulse-response backscattering, without the disadvantages of an intrusive technique.

\section{References}

[1] H. Schmuck, J. Hehmann, M. Straub and Th. Pfeiffer, "Embedded OTDR techniques for cost-efficient fibre monitoring in optical access networks", in Proc. (on CD-ROM) of the ECOC'06 (Sep. 2006).

[2] J. Bauwelinck, W. Chen, D. Verhulst D, Y. Martens, P. Ossieur, X.Z. Qiu and J. Vandewege, "A high-resolution burst-mode transmitter with fast and accurate optical level monitoring for a 1.25-Gb/s upstream PON", IEEE JSSC 40, 1322-1330 (Jun. 2005).

[3] D. Verhulst, Y.C. Yi, X.Z. Qiu, S. Verschuere, Z. Lou, P. Ossieur, J. Bauwelinck, X.Yin, and J. Vandewege "Upstream experiments on the gigabit PON physical medium layer", in Proc. (on CD-ROM) of the OFC'05 (Mar. 2005).

[4] B. De Mulder, W. Chen, J. Bauwelinck, J. Vandewege and X.Z. Qiu, "Non-Intrusive Fiber Monitoring of TDM Optical Networks", to be published by IEEE JLT. 